\title{
NEUMATOSIS INTESTINAL Y ALERGIA A LA PROTEINA DE LA LECHE DE VACA
}

\author{
Drs. Georgette Pose L, María Eugenia Arancibia S, Gloria Soto G, Aníbal Espinoza G, Isabel Fuentealba T, \\ Carmen G. Reginensi $R$.
}

Servicio de Diagnóstico por Imágenes y Pediatría, Clínica Alemana. Santiago, Chile.

\section{PNEUMATOSIS INTESTINALIS AND COW'SMILK PROTEINE ALLERGY}

\begin{abstract}
Pneumatosis intestinalis in newly born infants constitutes a clinical manifestation greatly associated with the presence of necrotizing enterocolitis, a highly severe medical condition. This study provides a clinical case series presenting with bloody stools and benign pneumatosis intestinalis. A short colonoscopy proven diagnosis of allergic colitis was made, which was confirmed in the $87,5 \%$ (7/8) of the cases, while a number of biopsies showed eosinophiles exceeding the normal ranges. Diagnosis in $12,5 \%$ of patients (1/8) was based on clinical records, lab-tests, imaging and treatment responses. The suppression of cow's milk protein in the maternal diet or the replacement of human milk by a substitute free from that protein led to a rapid regression of symptomatology.
\end{abstract}

Key words: Pneumatosis, necrotizing enterocolitis.

Resumen: La neumatosis intestinal en el recién nacido es un signo que se asocia mayoritariamente a la presencia de enterocolitis necrotizante, una condición grave. Se presenta una serie de casos clínicos que debutaron con sangre en la deposición y neumatosis intestinal de curso benigno, diagnosticándose una colitis alérgica, que fue demostrada en el 87,5\% (7/8) de los casos mediante colonoscopía corta y biopsias que demostraron un número de eosinófilos mayor que lo normal; en el 12,5\% (1/8) el diagnóstico se basó en clínica, laboratorio, imágenes y respuesta a tratamiento. La supresión de la proteína de la leche de vaca en la dieta de lamadre o el reemplazo de la leche materna por un sustituto libre de esta proteína, llevó a una rápida regresión de la sintomatología.

Palabras clave: Enterocolitis necrotizante, Neumatosis.

\section{Introducción}

La neumatosis intestinal en recién nacidos ( $R N)$ y lactantes es un signo que se asocia a enterocolitis

Pose G y cols. Neumatosis intestinal y alergia a la proteina de la leche de vaca. Rev Chil Radiol 2008; 14: 68-72. Correspondencia: Dra. Georgette Pose L. gpose@alemana.cl necrotizante (ECN), una condición grave en el RN. Esta se relaciona en la mayoría de los casos (85\%) con prematuros de menos de 35 semanas de gestación, bajo $2.500 \mathrm{~g}$ y muchas veces con otras patologías como enfermedad de Hirschsprung y cardiopatías congénitas complejas, en un contexto de isquemia, infección y signos de abdomen agudo ${ }^{(1,2)}$.

La clínica de la ECN con neumatosis intestinal (NI) aparece a menudo a los 3 ó 4 días de vida con sangre en deposiciones, vómitos, distensión abdominal, signos de sepsis y apnea, o a veces letargia. La ECN se ha asociado a hipoxia, estrés, hipotensión e infección ${ }^{(1)}$. La fisiopatología implica una isquemia intestinal por hipoperfusión de la pared y la subsiguiente caída de la barrera mucosa que permite la entrada de bacterias y/o aire a la pared intestinal, que puede ser causada o facilitada por necrosis de la pared intestinal o autolisis de la flora intestinal. El aire intersticial de la pared del intestino puede entrar a la circulación venosa portal, acompañado de acidosis y coagulación intravascular diseminada ${ }^{(3-5)}$.

Los hallazgos imaginológicos de ECN en la radiografía (Rx) de abdomen simple son inespecíficos. Entre los signos clásicos, los más frecuentemente observados son la distensión aérea difusa del intestino y la NI, que puede ser local o difusa, afectando frecuentemente el intestino delgado distal y colon derecho ${ }^{(1)}$.

Se han descrito otras causas de neumatosis que pueden simular $\mathrm{ECN}^{(6)}$ :

- Cateterización de la vena umbilical con producción de microémbolos retrógrados ${ }^{(7)}$

- Cambios hemodinámicos con isquemia intestinal, en ausencia de infección

- Neumomediastino, en el que el mecanismo postulado es la disección del aire desde el mediastino -a lo largo de la aorta y sus tributariasa las paredes intestinales

- Distensión en obstrucciones intestinales en el período de RN, como atresia duodenal, ano imperforado, íleo meconial y síndrome de tapón meconial, entre otros. Esta neumatosis puede desarrollarse por el escape de gas intestinal a través de ruptura de la mucosa con la subsiguiente 
disección submucosa y/o subserosa

- Isquemia de la pared intestinal asociada a una marcada sobredistensión del intestino

Nuestro objetivo es comunicar el aspecto imaginológico observado en la evolución de RN con alergia a la proteína de la leche de vaca, con especial énfasis en la presencia de neumatosis, debido a la escasa información existente en la literatura en relación con este tema.

\section{Material y método}

Se revisaron retrospectivamente las fichas clínicas de una serie de $8 \mathrm{RN}$ con alergia a la proteína de la leche de vaca, demostrada mediante colonoscopía corta y estudio histológico, con recuento absoluto de eosinófilos por campo de aumento mayor y por diez campos. Se consideró colitis alérgica la presencia de más de 20 eosinófilos por campo de aumento mayor o más de 60 eosinófilos en 10 campos de aumento mayor, criterio usado en el Hospital de Niños de Filadelfia CHP.

Se consignaron los antecedentes perinatales, estudio imaginológico, métodos diagnósticos y respuesta al tratamiento, analizando los hallazgos y la relación entre los distintos parámetros.

\section{Resultados}

De los 8 pacientes estudiados, 3 correspondían a sexo femenino y 5 a masculino. La edad gestacional fluctuó entre las 34 y 38 semanas de vida (mediana de 37 semanas), con peso al nacer entre $2.430 \mathrm{~g} \mathrm{y}$ $4.125 \mathrm{~g}$ (mediana de $2.875 \mathrm{~g}$ ).

En el $75 \%$ (6/8) de los pacientes existió una o más patologías asociadas, que se describen en la Tabla I.

\begin{tabular}{|ll|}
\hline Tabla I. Morbilidad asociada. \\
\hline Hiperbilirrubinemia & 1 caso \\
Hipoglicemia & 3 casos \\
Hernia diafragmática & 1 caso \\
Infarto Silviano & 1 caso \\
\hline
\end{tabular}

En el $87,5 \%$ (7/8) de los RN estudiados, el motivo de consulta y alerta fue la presencia de sangre en la deposición, asociada a dolor cólico en un $50 \%$ (4/8) de los casos, vómitos en 12,5\% (1/8), cólico más distensión en $12,5 \%$ (1/8) y dolor cólico más constipación en $12,5 \%$ (1/8).

La mitad de los pacientes (4/8) estaban hospitalizados al momento de presentar los síntomas: 2 por parto gemelar con hipoglicemia transitoria, 1 por apnea a las 48 horas de vida y 1 por arritmia. Los restantes cuatro pacientes consultaron ambulatoriamente.
La sintomatología se presentó entre las 24 horas y los 38 días de vida (mediana de 16, 9 días). La alimentación en el momento del diagnóstico fue: pecho exclusivo en el $50 \%$ ) pecho más fórmula en $37,5 \%$ y sólo fórmula en el $12,5 \%$ de los niños.

Al examen físico destacaba ausencia de compromiso hemodinámico y examen clínico abdominal normal en la mayoría de los pacientes (7/8); sólo un paciente presentó distensión abdominal.

Se descartó abdomen agudo y patología infecciosa en todos los RN, tanto clínicamente como con exámenes de laboratorio.

Los hallazgos de la $\mathrm{Rx}$ de abdomen simple se describen en la tabla II. La neumatosis intestinal (NI) se observó radiológicamente en siete pacientes $(87,5 \%)$ (Figura 1). En un caso la Rx no mostró alteraciones, observándose aire portal en el ultrasonido (US) (Figura 2).
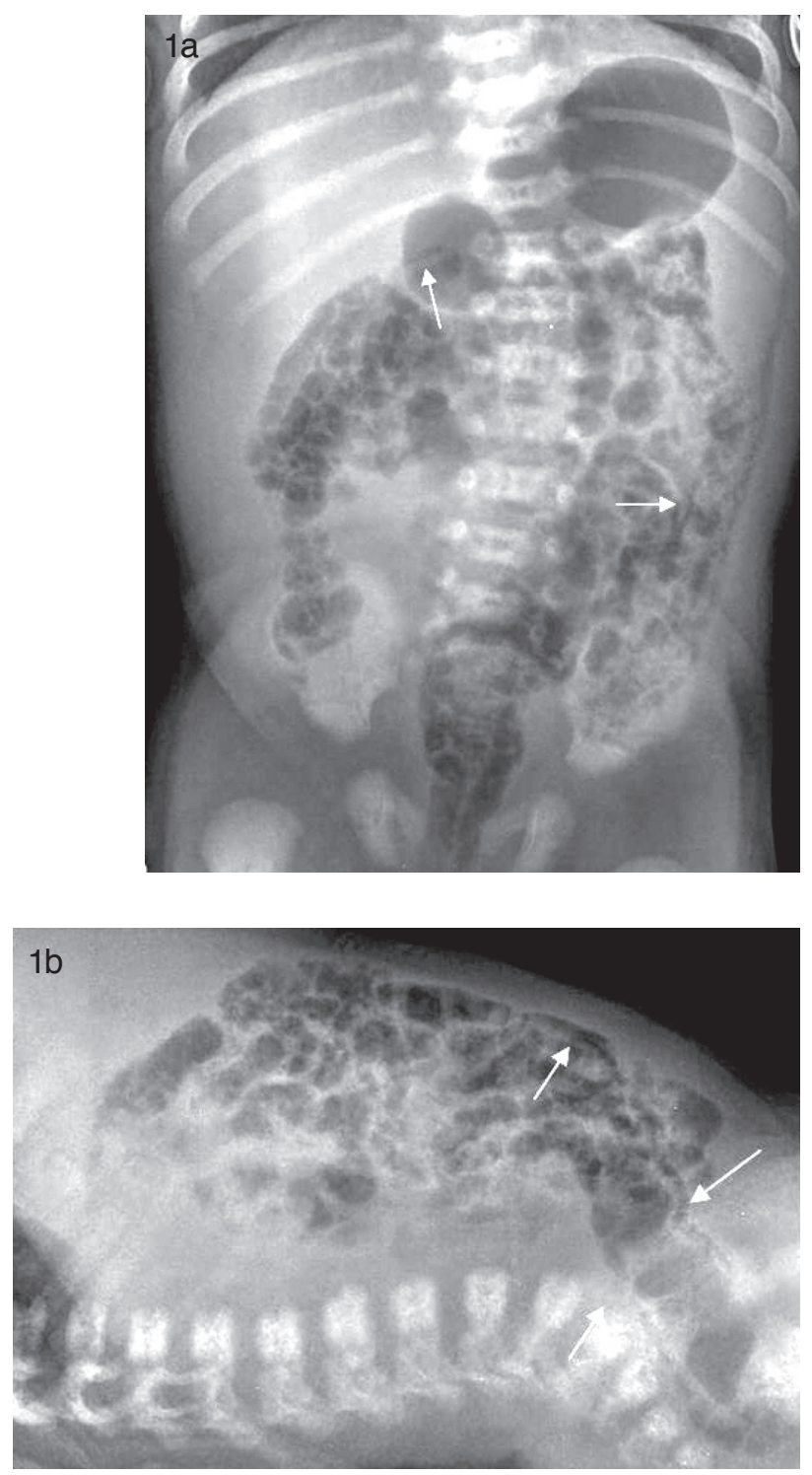

Figura 1. Extensa neumatosis en colon ascendente, transverso, descendente y recto, sin distensión intestinal. a) $R x A P$ y b) $R x$ lateral, (Caso 5). 

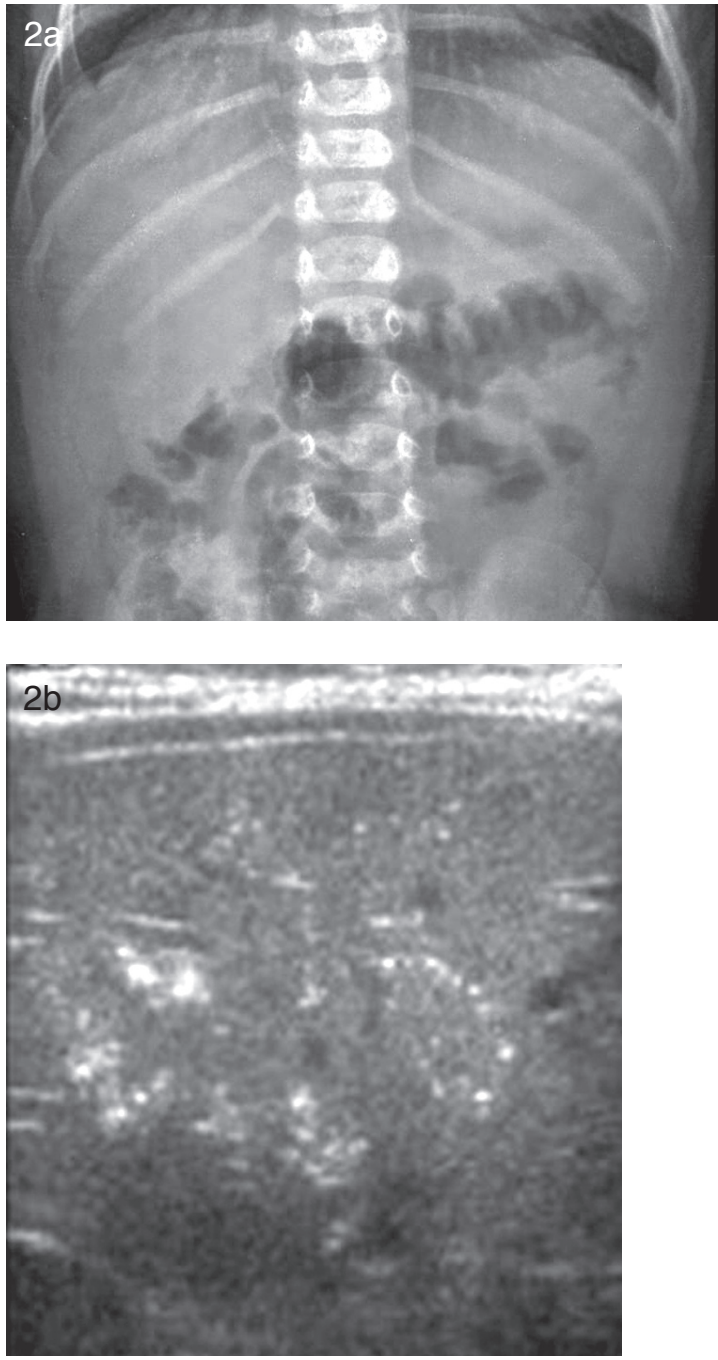

Figura 2. $R x$ de abdomen simple sin alteraciones y b) aire portal en US (Caso 7).

La NI se localizó principalmente en el colon izquierdo (Figura 3) y a nivel de colon derecho (Figura 4) y transverso (Figura 5) Asociada a NI se observó también engrosamiento de paredes intestinales (Figura 6) y solamente en un paciente existió distensión intestinal (Figura 7).

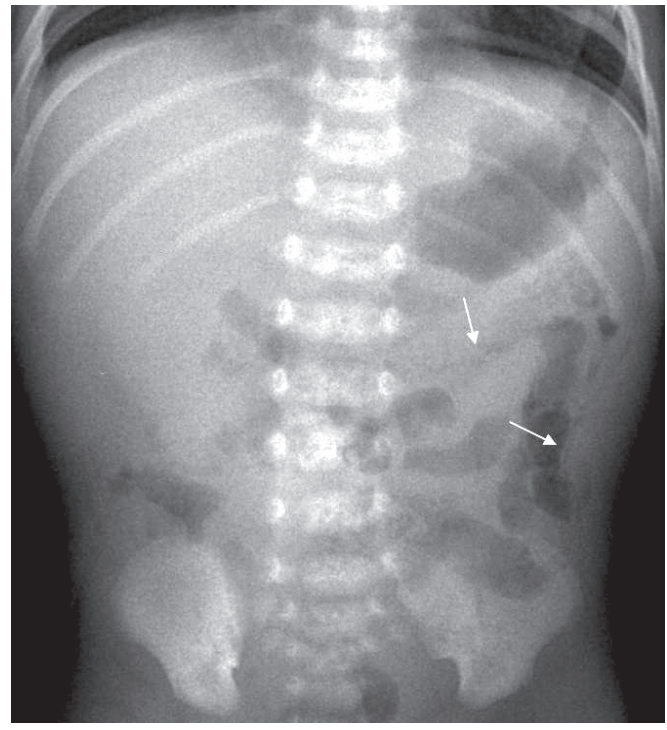

Figura 3. Neumatosis en colon descendente, y transverso sin distensión intestinal (Caso 6).

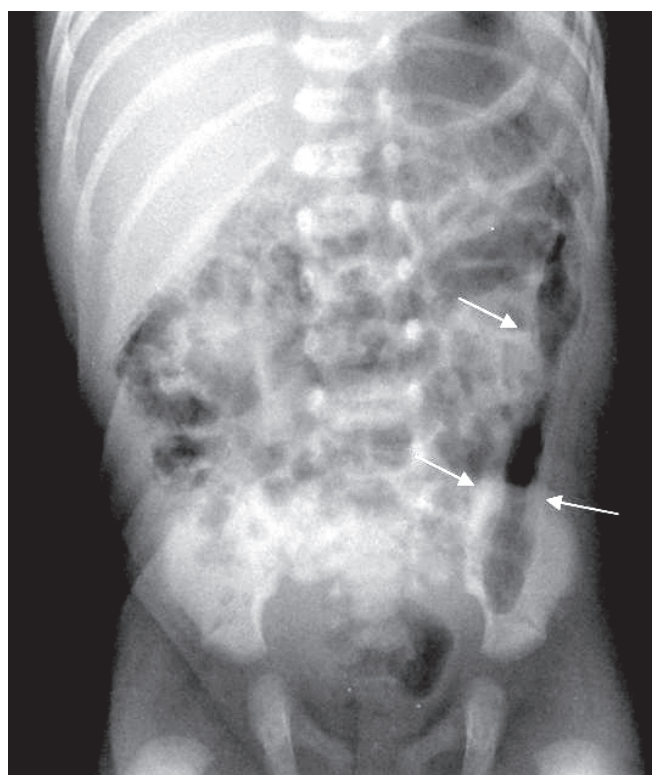

Figura 4. Neumatosis en colon derecho e izquierdo, asociada a marcado engrosamiento parietal a izquierda (flechas) (Caso 4).

Tabla II. Hallazgos imaginológicos

Neumatosis leve(+), moderada)(++), severa(+++)

\begin{tabular}{|l|c|c|c|c|c|c|c|c|}
\hline & $\begin{array}{c}\text { Caso } \\
\mathbf{N}^{\circ} \mathbf{1}\end{array}$ & $\begin{array}{c}\text { Caso } \\
\mathbf{N}^{\circ} \mathbf{2}\end{array}$ & $\begin{array}{l}\text { Caso } \\
\mathbf{N}^{\circ} \mathbf{3}\end{array}$ & $\begin{array}{c}\text { Caso } \\
\mathbf{N}^{\circ} \mathbf{4}\end{array}$ & $\begin{array}{c}\text { Caso } \\
\mathbf{N}^{\circ} \mathbf{5}\end{array}$ & $\begin{array}{l}\text { Caso } \\
\mathbf{N}^{\circ} \mathbf{6}\end{array}$ & $\begin{array}{c}\text { Caso } \\
\mathbf{N}^{\circ} \mathbf{7}\end{array}$ & $\begin{array}{l}\text { Caso } \\
\mathbf{N}^{\circ} \mathbf{8}\end{array}$ \\
\hline Neumatosis colon der & - & - & - & + & ++ & - & - & ++ \\
Neumatosis colon transv. & - & + & + & - & +++ & + & - & - \\
Neumatosis colon izq/sigm & + & ++ & + & + & +++ & + & - & - \\
Neumatosis int delgado & - & - & - & - & - & - & - & - \\
Engrosamiento colon der. & - & - & - & - & - & - & - & - \\
Engrosamiento colon izq. & +++ & + & - & +++ & + & + & - & - \\
Distensión & - & - & + & - & - & - & - & - \\
Aire portal & - & - & - & - & - & - & $+($ US $)$ & - \\
\hline
\end{tabular}



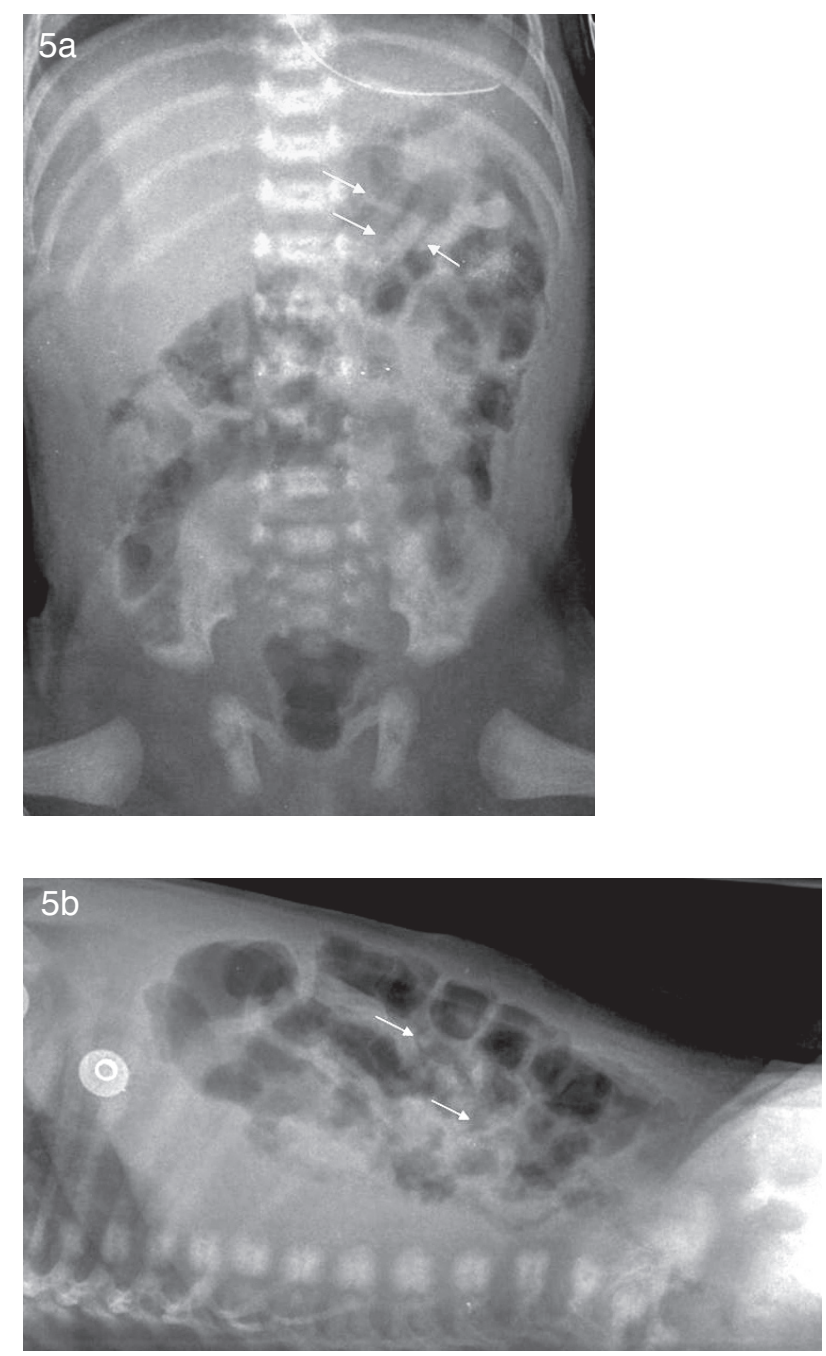

Figura 5. Neumatosis en colon transverso y descendente, sin distensión intestinal significativa. a) $R x A P$ y b) $R x$ lateral, (Caso 2).

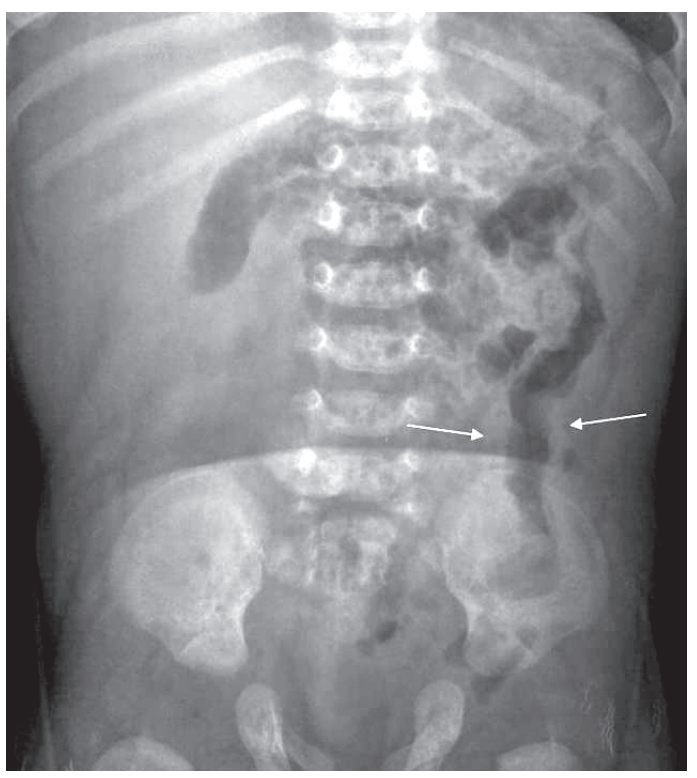

Figura 6. Engrosamiento de las paredes del colon izquierdo asociada a neumatosis, sin distensión intestinal. (Caso 1).
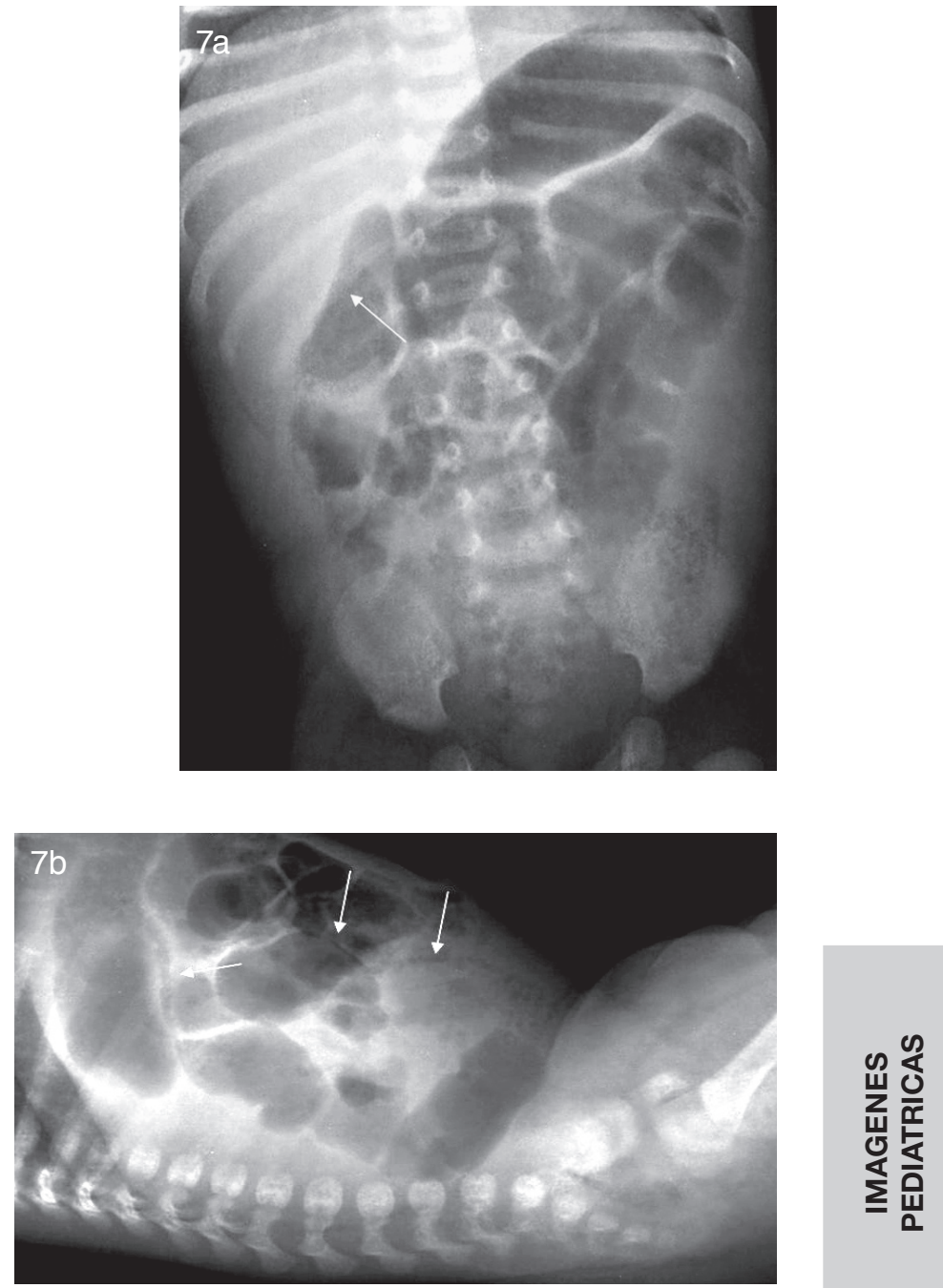

Figura 7. Neumatosis en colon sigmoides y transverso, asociada a distensión intestinal. a) $R x A P$ y b) $R x$ lateral, (Caso 3).

Un paciente presentó constipación antes de la rectorragia, por lo que se estudió con enema baritada, que resultó normal, y manometría rectal, que demostró hipertonía, por lo que se le realizó una esfinteromiotomía cuya biopsia fue negativa para enfermedad de Hirschsprung.

Se efectuó colonoscopía en 7 de 8 pacientes, demostrándose colitis de aspecto alérgico, que fue confirmada histológicamente mediante biopsia. La biopsia del paciente que presentaba aire portal sin neumatosis radiológica resultó concordante con colitis alérgica, por lo que se trató de la misma manera que el resto de los pacientes, con supresión de la proteína de la leche de vaca en la dieta materna en los niños alimentados al pecho exclusivo o, el reemplazo de la fórmula por un sustituto libre de esta proteína. Uno de los últimos pacientes de esta serie, que presentaba 
sangre en deposiciones y neumatosis radiológica con clínica y de laboratorio benignos, se trató empíricamente como colitis alérgica.

La evolución en todos los pacientes fue favorable, con regresión de la sintomatología y de la neumatosis en las primeras 48 horas, desde el inicio del tratamiento.

\section{Discusión}

En la evolución de los niños de esta serie que presentaron $\mathrm{Nl}$ en la Rx simple, llama la atención que todos eran $\mathrm{RN}$ de más de 34 semanas de gestación, adecuados para la edad gestacional y con Apgar normal, en los que se descartó un abdomen agudo y los exámenes de laboratorio excluyeron infección, todo lo cual hacía altamente improbable una ECN.

La neumatosis en ECN afecta con mayor frecuencia el ciego y habitualmente se asocia a distensión de asas, lo que contrasta con la afectación radiológica observada en este grupo, que correspondió mayoritariamente a colon izquierdo y se presentó con signos de engrosamiento de paredes intestinales, separación entre asas adyacentes, ausencia de haustras y escasa distensión intestinal en la mayoría de los pacientes.

EI US se ha constituido en una excelente herramienta diagnóstica complementaria en la pesquisa de neumatosis, gas portal y estudio de las paredes intestinales ${ }^{(2,8)}$, como se comprobó en uno de nuestros pacientes en que detectamos el gas portal sólo mediante este método.

Pensamos que la etiología de esta neumatosis probablemente se relacione con un proceso inflamatorio no infeccioso que altere la permeabilidad de la pared y permita el escape de aire desde la mucosa. El factor de distensión intestinal, presente en la ECN, no fue un elemento importante en nuestro grupo ya que en esta alteración radiológica estuvo presente sólo en un paciente.

\section{Conclusiones}

La neumatosis intestinal se observa como signo radiológico en $\mathrm{RN}$ con colitis alérgica.

Debe considerarse la alergia a la proteína de la leche de vaca como posibilidad diagnóstica en todo RN de término que se presenta con rectorragia y neumatosis, en ausencia de abdomen agudo o infección.

\section{Bibliografía}

1. Daneman A, Woodward S, de Silva M. The radiology of neonatal necrotizing enterocolitis (NEC). A review of 47 cases and the literature. Pediatr Radiol 1978; 7: 70- 77.

2. Epelman M, Daneman A, Navarro O. Necrotizing Enterocolitis: Review of State-of the-Art: Imaging Findings with Pathologic Correlation. Radiographics 2007; 27: 283-305.

3. Kogutt MS. Necrotizing enterocolitis of infancy. Early roentgen patterns as a guide to prompt diagnosis. Radiology 1979; 130: 367-370.

4. Leonidas JC, Hall RT, Amoury RA. Critical evaluation of the roentgen signs of neonatal necrotizing enterocolitis. Ann Radiol 1976; 19: 123-132.

5. Wexler HA. The persistent loop sign in neonatal necrotizing enterocolitis: a new indication for surgical intervention? Radiology 1978; 126: 201-204.

6. Robinson AE, Grossman H, Brumley GW. Pneumatosis intestinals in the neonate. Am J Roentgenol Radium Ther Nucl Med 1974; 120(2): 333-341.

7. Coussement AM, Gooding CA, Taybi H, Faure CC. Roentgenographic visualization of the umbilical arteries in pneumoperitoneum in the newborn. AJR 1973; 118: 46-48.

8. Merritt CR, Goldsmith JP, Sharp MJ. Sonographic detection of portalvenous gas in infants with necrotizing enterocolitis. AJR 1984; 143: 1.059-1.062. 